\title{
Prevención de violencia sexual mediante la investigación como estrategia pedagógica en educación básica ${ }^{1}$ Prevention of sexual violence through research
as a pedagogical strategy in basic education
}

DOI: http://dx.doi.org/10.17981/cultedusoc.9.1.2018.15

Fecha de recepción: 08/05/2018. Fecha de aceptación: 09/08/2018

Yudy Cardona-Jaramillo²; Belkys Varela-Suarez ${ }^{3}$

Rosa María Morales-Ibáñez; Flavia Inés Mercado-Sanjuán; Olfa Esther Cantillo-Mercado; Manuela Hernández-Pedroza; Olga Marina Villazon-De Jinete; Ibeth Altahona-Mercado; Bibiana Concepción De La Rosa-De Polo; Nadime Isabel Fadul-Sanjuán ${ }^{4}$

Para citar este artículo

Cardona-Jaramillo, Y., Varela-Suarez, B., Morales-Ibáñez, R., Mercado-Sanjuán, F., Cantillo-Mercado, O., Hernández-Pedroza, M., Villazon-De Jinete, O., Altahona-Mercado, I., De La Rosa-De Polo, B. y Fadul-Sanjuán, N. (2018). Prevención de violencia sexual mediante la investigación como estrategia pedagógica en educación básica. Cultura. Educación y Sociedad 9(1), 194-207. DOI: http://dx.doi.org/10.17981/cultedusoc.9.1.2018.15

\section{Resumen}

La violencia sexual es una problemática de salud pública que viene en aumento, convirtiéndose en un flagelo nacional, presentándose especialmente al interior del núcleo familiar, pero se manifiesta también en las diversas instituciones del colectivo social. El estudio busco prevenir la violencia sexual mediante la investigación como estrategia pedagógica en educación básica. Se guio bajo el enfoque de Investigación como estrategia pedagógica. Donde se utilizaron herramientas como diario de campo y observación participante. La unidad de análisis estuvo conformada por cuarenta (40) estudiantes de la IED Francisco de Paula Santander, Sede Simón Bolívar de Fundación-Magdalena. Los resultados evidenciaron que los alumnos no tenían conocimientos previos sobre violencia sexual, luego de la integración de la IEP al aula de clase desde el área de sociales se logró la apropiación y socialización de los conocimientos adquiridos a través del proceso de indagación por parte de los estudiantes con el apoyo docente.

Palabras clave: prevención de violencia sexual, investigación como estrategia pedagógica, educación básica.

\begin{abstract}
Sexual violence is a problem of public health that is increasing, becoming a national scourge, appearing especially within the family nucleus, but also manifested in the various institutions of the social collective. The study sought to prevent sexual violence through research as a pedagogical strategy in basic education. It was guided under the Research approach as a pedagogical strategy. Where tools were used as field diary and participant observation. The analysis unit consisted of forty (40) students of the IED Francisco de Paula Santander, Simón Bolívar campus of Fundación- Magdalena. The results showed that the students did not have previous knowledge about sexual violence, after the integration of the IEP into the classroom from the social area, the appropriation and socialization of the acquired knowledge was achieved through the process of inquiry by the students. students with teacher support.
\end{abstract}

Keywords: prevention of sexual violence, research and pedagogical strategy, basic education.

\footnotetext{
${ }^{1}$ Este artículo ha sido derivado del Programa de Fortalecimiento de la Cultura Ciudadana y Democrática CT+I a través de la IEP apoyada en TIC en el Departamento de Magdalena: CICLON

${ }^{2}$ Psicóloga especialista en Psicoterapia Infantil de la Universidad de la Costa, CUC. Correo de correspondencia: yudycardonaj@ gmail.com

${ }^{3}$ Docente de la Institución Educativa Departamental Francisco de Paula Santander. Líder del grupo de investigación Transformadores de la Comunidad. Correo de correspondencia: belk@hotmail.com.

${ }^{4}$ Docentes de la Institución Educativa Francisco de Paula Santander, Sede Simón Bolívar y miembros del grupo de investigación Transformadores de la Comunidad.
}

- The author; licensee Universidad de la Costa - CUC. Cultura, Educación y Sociedad vol. 9 no. 1, pp. 194-207. Enero - Junio, 2018 Barranquilla. ISSN 2389-7724 Online 


\section{Introducción}

El abuso sexual es una temática que se ha trabajado de forma ardua desde distintos ámbitos estatales, ya que a pesar de la existencia de diversas campañas en las que se involucra a niños en el conocimiento del abuso sexual, este fenómeno sigue creciendo en los distintos países. Impactando de forma negativa las sociedades ya que no solo involucra el daño en el desarrollo sexual del niño sino además es una forma de maltrato infantil, en el que intervienen otros componentes importantes que hacen parte del libre y sano desarrollo del niño. En otras palabras, los mecanismos de arbitrariedad más importantes que afectan a las mujeres en el sistema educativo ya no se sitúan en el acercamiento al sistema, sino en la calidad y en las modalidades de enseñanza, lo que impide una justicia real de oportunidades entre los sexos.

En referencia a la temática seleccionada, la nación unida en el documento de la UNICEF lo considera como el "crimen encubierto más extendido de la humanidad". En este mismo orden de ideas, lo define como el acto de involucrar a niños, niñas y adolescentes en actividades sexuales de cualquier índole con o sin contacto corporal y con o sin violencia física, en las que el agresor busca la gratificación personal, sexual y la víctima padece abuso de fuerza y de poder por la asimetría natural de desarrollo. En ese mismo sentido, de acuerdo a las distintas áreas de la salud la violencia sexual está reconocida como un factor de riesgo para la salud mental, reportándose una diversidad de síntomas asociados a estas experiencias de vulneración, entre ellos: estrés postraumático, depresión, bajo desempeño académico, agresividad, aislamiento y conductas irritantes, (Pereda, 2009).
De acuerdo a los razonamientos que se han venido realizando, la temática es compleja y abarca múltiples variables que aportan un nivel más alto de gravedad en la persona que lo padece, en este propósito Guerra, Ocaranza, y Weinberger (2016), plantean que la variabilidad en las consecuencias de la violencia sexual infantil y adolescentes, ha sido explicada en función de una serie de factores directamente vinculados a la gravedad del abuso sexual como el nivel de contacto físico de la agresión, la relación de la víctima con el agresor, el tiempo que ha pasado desde la agresión y la existencia de otras vulneraciones presentes en la víctima además del abuso.

Es de suma importancia considerar que el abuso sexual es un tema social en el que se deben tomar medidas necesarias para evitar que se siga manifestando. En este orden de ideas, se hace necesario trabajar de forma incisiva en la temática ya que una de las principales causas de su presentación es el desconocimiento de los niños hacia el significado del término abuso sexual y los posibles comportamientos que pudieran manifestarse dando como resultado una conducta de riesgo.

Por consiguiente, cuando se presenta un abuso sexual las consecuencias para el niño pueden marcar su vida adulta, comportamientos con su pareja, de igual forma manifestarse en su salud psicológica, afectando su interacción con el medio en cuanto a las relaciones sociales, sus relaciones familiares se generarían un agravante en el caso de que el abuso sea cometido por un familiar. De igual forma, en el contexto académico el nivel de riesgo es igual que en los escenarios anteriores, por tal razón, el reconocimiento por parte de los niños es importante trabajarlo desde edades tempranas. 
Con referencia a loa anterior, es de suma importancia promover actividades que originen conocimientos para prevenir un posible abuso sexual en los estudiantes, teniendo como base el reconocimiento de su cuerpo hasta llegar a la forma como se dan las relaciones sociales del entorno en el cual se desenvuelven. Este plan de actividades siendo orientado por los docentes co-investigadores, sin embargo, son los estudiantes quienes trabajaran en las estrategias orientadas a mejorar sus conocimientos sobre sexualidad, buscando generar aprendizaje en los niños, en educación sexual para evitar conductas de riesgo que los haga víctimas de un posible abuso.

El objetivo del estudio es prevenir la violencia sexual mediante la IEP en los niños y niñas de la IED Francisco de Paula Santander, sede Simón Bolívar, además, se hará uso de las TIC para generar actitudes reflexivas en torno a la percepción de la situación problema en todos los niveles de la sociedad colombiana; de igual manera se espera que este estudio se convierta en referente teórico práctico sobre la problemática planteada en busca de alternativas favorables a la solución de este conflicto.

\section{Elementos teóricos de la violencia sexual mediante la IEP en la escuela}

En efecto, el contexto escolar constituye uno de los espacios que más poderosamente eficaz en la construcción de la identidad personal de hombres y de mujeres, y de su futuro proyecto de vida. En cada enlace social se construye un conjunto de rasgos de pensamiento, de valoraciones, de afectos, de actitudes $\mathrm{y}$ de comportamientos, que se asumen como típicos y como referentes del deber ser y de pertenencia, según se sea hom- bre o mujer. Estos rasgos que revelan la identidad de las personas, contienen a la vez elementos asociados a los atributos, a los roles, a los espacios de actuación, a los derechos y obligaciones y a las relaciones de género. Se plantean de manera explícita a través de propuestas educativos, mediante normativas de reglamentación, es decir, parten del discurso de la institución escolar, o, por lo general, son fragmentos del currículo oculto o escondido.

En el campo de las interacciones en la escuela, esta debe ser vista como una agencia socializadora, compleja, dinámica y coherente, que involucre la comunidad general, en la que convivan en tensión representación de géneros diversas; como un ámbito de lucha, de resistencia y de creación de normativas, valores, practicas legítimas, normales y transgresoras. Gómez y Cobo, (2013) definen el abuso sexual, como la utilización de un niño en actividades sexuales que él no está en capacidad de comprender, para las que no está física, ni mentalmente preparado y, por tanto, no puede dar su consentimiento; violando la ley o las normas sociales con la finalidad de satisfacer o gratificar sexualmente a un adulto o grupo de personas adultas

La violencia sexual también se presenta en ámbitos escolares, sin embargo, los abusos más graves suceden dentro del seno familiar, repercutiendo esto de forma radical en las conductas de niños y adolescentes que padecen esta situación. Las secuelas psicológicas afectarían de forma directa su desarrollo psicosexual, moral, afectivo y social. En ámbitos como en el familiar y personal las consecuencias son en su gran mayoría permanentes.

El sistema escolar normalmente proporciona al estudiante un espacio para 
que establezca relaciones de proximidad con su grupo de pares y docentes. Además, es un escenario donde se realizan actividades preventivas frente a las situaciones de abuso, entregando información que permite al estudiante discriminar entre lo legítimo o no de estos actos. El medio escolar puede representar para el niño abusado la primera oportunidad de hablar de esta experiencia. Sin embargo, en algunos casos es en el colegio donde se lleva a cabo el abuso, ya sea por profesores, estudiantes de grados superiores, personal administrativo o de servicio, provocando daño psicológico al menor, por la transformación de un medio protector en un medio agresor y amenazante, (Almonte, Insunza, y Ruiz, 2002).

En este sentido el Fondo de las Naciones Unidas para la infancia (UNICEF, 2013) informa que en la mayoría de los casos el abuso sexual infantil es cometido por un familiar o persona de confianza del niño. "La dimensión y la gravedad de esta forma de violencia ejercida contra la infancia vuelven sumamente relevante el diseño de políticas públicas que promuevan la prevención, la recolección de datos y la identificación de las víctimas de abuso sexual.

Asimismo, son necesarias campañas de sensibilización dirigidas tanto a los niños y adolescentes como a los adultos que se desempeñan en los sistemas de protección de derechos, salud, educación, policía, justicia y a la sociedad en general. Sin detección no es posible implementar medidas de protección, ni brindar tratamiento para las víctimas y sus familias. Al mismo tiempo, debemos considerar que el agresor sexual que no reconoce su crimen, que no busca tratamiento, que no es identificado ni recibe sanción alguna representa un riesgo para los niños $\mathrm{y}$ para toda la sociedad.
De igual forma se debe tener en cuenta que no solo los niños y adolescentes que viven con sus familiares se encuentran en riesgo hay otro tipo de situaciones que los colocan en punto vulnerable como: historial de abandono, baja autoestima y antecedentes de ser mal tratados ya que son accesibles y fáciles de engañar. Resulta oportuno resaltar a la familia como primer grupo en el que se desarrolla el individuo y su papel en la educación sexual para prevenir el abuso, en este orden de ideas

Miranda (2014), sugiere que los padres deben permitir el conocimiento de la información básica materializándolas en el desarrollo de sus emociones, vivencias y cambios y de forma cíclica abordar los conocimientos del cuidado del cuerpo, de los cambios que ocurren, la integración de esos cambios en el desarrollo de la personalidad, en las buenas prácticas de la salud y la prevención de la violencia sexual. Para que estos espacios se realicen las familias deben desarrollar espacios positivos de diálogos habilidades técnicas para conversar las veces que sean requeridas con los niños. Con esta respuesta activa por parte de los padres no solo se busca que impacten de forma positiva los conocimientos sexuales de sus hijos si no que se afiancen los vínculos afectivos $\mathrm{y}$ con ellos se nutran las habilidades para la socialización dentro de la familia como fuera de ella.

En este sentido el desarrollo sexual del individuo se verá marcado por las habilidades que le permitan socializarse mejor con el medio y de esta forma su sexualidad estará bajo criterios como la solidaridad, el amor, el respeto hacia la identidad del otro y asumiendo actitudes de responsabilidad hacia la sexualidad. Las consecuencias del abuso sexual en la niñez implican un impacto a nivel social. 
Pereda (2010) realizó una revisión de las consecuencias psicológicas a largo plazo en víctimas de abuso sexual infantil, resaltando cinco categorías: Problemas emocionales, problemas de relación, problemas funcionales, problemas de adaptación, y problemas sexuales; lo cual requiere de docentes profesionales que trabajen en un trabajo coordinado en el tema de la educación sexual y coopere en la presentación de estos problemas.

Los docentes formamos parte activa de la vida del niño, compartimos espacios de tiempo y desarrollamos con ellos actividades de correspondientes a la academia, en este orden de ideas somos gestores de la forma en que elaboren su actividad social y familiar, específicamente mediamos en los comportamientos que el niño demuestre en estos espacios, de acuerdo con este razonamiento Morillo, B. (2012) señala la importancia de poner en práctica estrategias educativas que permitan la prevención del abuso sexual infantil y que sean los docentes quienes contribuyan a combatir este flagelo social. De acuerdo a este razonamiento realizado por el autor los docentes estamos obligados a trabajar con el alumno en la especificidad de este tema teniendo en cuenta objetivos educacionales que estén secuenciados desde lo más simple a lo más complejo desarrollando un sistema de clasificación de objetivos en tres aspectos: el cognitivo, el afectivo y el psicomotor.

De acuerdo con Meresman (2011), la sexualidad humana se define como: "Un aspecto central del ser humano, presente a lo largo de su vida. Abarca al sexo, las identidades y los papeles de género, el erotismo, el placer, la intimidad, la reproducción y la orientación sexual. Se vivencia y se expresa a través de pensamientos, fantasías, deseos, creencias, actitudes, valores, conductas, prácticas, papeles y relaciones interpersonales".

Anceschi (2009), señala que la violencia es un concepto subjetivo de definición compleja, ya que puede adquirir diversos tipos de acepciones según el punto de vista desde el que lo analicemos. Así la definición no será la misma desde una perspectiva moralista o jurídica y dentro del ámbito jurídico un penalista no la definirá de la misma manera que un civilista. Es por ello que realmente su definición es compleja identificando casos en los que incluso esta es "legítima" como método de resolución de conflictos.

Así mismo, Anceschi (2009), ninguna norma del ordenamiento italiano define el concepto de violencia en sentido general a excepción de la ley penal militar, la cual define solamente algunas de las formas más graves de violencia física aplicables al ámbito militar. La OMS (2016) define como todo acto que guarde relación con la práctica de la fuerza física o verbal sobre otra persona, animal u objeto originando un daño sobre los mismos de manera voluntaria o accidental. El elemento principal dentro de las acciones violentas, es el uso de la fuerza tanto física como psicológica para el logro de los objetivos, y en contra de la víctima.

Según la OMS (2016), la violencia sexual se define como: todo acto sexual, la tentativa de consumar un acto sexual, los comentarios o insinuaciones sexuales no deseados, o las acciones para comercializar o utilizar de cualquier otro modo la sexualidad de una persona mediante coacción por otra persona, independientemente de la relación de esta con la víctima, en cualquier ámbito, incluidos el hogar y el lugar de trabajo. La violencia sexual incluye la violación, definida como la penetración forzada físicamente o empleando otros medios de coacción, por 
más leves que sean, de la vulva o el ano, usando un pene, otras partes corporales o un objeto. El intento de realizar algunas de las acciones mencionadas se conoce como intento de violación. La violación de una persona llevada a cabo por dos o más agresores se denomina violación múltiple. La violencia sexual puede incluir otras formas de agresión que afecten a un órgano sexual, con inclusión del contacto forzado entre la boca y el pene, la vulva o ano.

Cabe decir que, los actos de violencia sexual pueden ser muy variados $\mathrm{y}$ producirse en circunstancias y ámbitos muy distintos. Entre ellos, cabe señalar que la infracción en el matrimonio o en las citas amorosas; la violación por parte de desconocidos; la violación sistemática durante los conflictos armados; las insinuaciones o el acoso no deseados de carácter sexual, con inclusión de la exigencia de sostener relaciones sexuales a cambio de favores; el abuso sexual de personas física o mentalmente discapacitadas; abuso sexual de menores; matrimonio o la cohabitación forzados, incluido el casamiento de menores; denegación del derecho a hacer uso de la anticoncepción o a adoptar otras medidas de protección contra las enfermedades de transmisión sexual; aborto forzado; actos de violencia que afecten la integridad sexual de las mujeres, incluida la mutilación genital femenina y las inspecciones obligatorias para comprobar la virginidad; la prostitución forzada y la trata de personas con fines de explotación sexual.

Según la ley colombiana las normas para la prevención de la violencia sexual y atención integral de los niños, niñas y adolescentes abusados establecen en sus artículos la siguiente definición para Violencia sexual en niños y niñas. Art. 2 Definición; Para efectos de la presen- te ley se entiende por violencia sexual contra niños, niñas y adolescentes, todo acto o comportamiento de tipo sexual ejercido sobre un niño, niña o adolescente, utilizando la fuerza o cualquier forma de coerción física, psicológica o emocional, aprovechando las condiciones de indefensión, de desigualdad y las relaciones de poder existentes entre víctima y agresor.

El Ministerio de Educación Nacional Colombiano la educación es un proceso de formación permanente, personal cultural y social que se fundamenta en una concepción integral de la persona humana, de su dignidad, de sus derechos y de sus deberes. En nuestra Constitución Política se dan las notas fundamentales de la naturaleza del servicio educativo. Allí se indica, por ejemplo, que se trata de un derecho de la persona, de un servicio público que tiene una función social y que corresponde al Estado regular y ejercer la suprema inspección y vigilancia respecto del servicio educativo con el fin de velar por su calidad, por el cumplimiento de sus fines y por la mejor formación moral, intelectual y física de los educandos.

También se establece que se debe garantizar el adecuado cubrimiento del servicio y asegurar a los menores las condiciones necesarias para su acceso y permanencia en el sistema educativo. El sistema educativo colombiano lo conforman: la educación inicial, la educación preescolar, la educación básica (primaria cinco grados y secundaria cuatro grados), la educación media (dos grados y culmina con el título de bachiller.), y la educación superior.

Por su parte las estrategias pedagógicas son todas las acciones realizadas por el docente, con el fin de facilitar la formación y aprendizaje de los estudian- 
tes. "Componen los escenarios curriculares de organización de las actividades formativas y de interacción del proceso enseñanza-aprendizaje donde se logran conocimientos, valores, prácticas, procedimientos y problemas propios del campo de formación", (Bravo, 2008).

En la actualidad, existen propuestas metodológicas de enfoques basados en investigación, entendidos como la modo de plasmar lo que se hace y como se implementa donde surgen inmensidad de propuestas metodológicas que se basan en un orientación o toman varios para hacer de ellos una pertinencia metodológica; es el caso de las pedagogías basadas o fundadas en investigación, las cuales se desarrollan en diferentes contextos y asumen variados caminos en conexión con los paradigmas y corrientes en los cuales se inscriben. Dentro de esta teoría basada en investigación se encuentra la Enseñanza por descubrimiento y el Programa Ondas, con su propuesta de la Investigación como Estrategia Pedagógica (IEP), como una perspectiva que se desarrolla en ese enfoque y toma elementos de la pedagogía critico-liberadora y del enfoque socio-cultural, (Mejía y Manjarrés, 2010).

Los contenidos del proceso educativo se convierten en problemas, y a partir de preguntas se estructura el currículo, en donde los avances y desarrollos de los estudiantes se determinan por la ampliación de estructura previa que se manifiesta en lenguaje, raciocinios y conocimientos. Ese descubrimiento no es necesariamente autónomo, sino que se hace guiado por el profesor, quien se encarga de planificar los ejercicios y los procedimientos pare el fin buscado (Pozo, 2006).

En igual forma, el eje de la propuesta es la idea de que en la sociedad existen saberes propios de la cultura, los cuales negocian invariablemente con las formas establecidas del conocimiento; por ello, la indagación planteada en los grupos infantiles y juveniles buscar la unidad y relación de saberes y conocimientos como partes complementarias, a través de una propuesta metodológica que realiza el reconocimiento social de los actores, quienes ponen en juego esas diferentes concepciones, que podrán tramitar buscando la visibilidad de múltiples métodos investigativos, en coherencia con el tipo de problema.

En tal sentido, la Investigación como Estrategia Pedagógica IEP da una nueva constitución de subjetividad de estos tiempos, donde las culturas infantiles y juveniles, en el sentido de ser nativos digitales, no solamente hacen su uso, sino que impugnan la desigualdad en su práctica pedagógica, interpelan la legitimidad de ella y construyen horizontes de posibilidad en donde su práctica grupal le muestra que hay futuro compartido y que él aquí y ahora, con esa colectividad con la que trabaja, lo está construyendo y le permite pensar esos cambios y esas transformaciones porque los comienza a vivir en su vida.

Además, el maestro y la maestra que trabajan con la IEP comprenden las posibilidades de esas evoluciones y se alimentan de un ejercicio que realizan con sus grupos para producir los cambios y transformaciones en la esfera de su práctica pedagógica, e inician la marcha no por una ley o un mandato, sino porque encuentran allí que pueden ser educadores de otra manera, sin miedo, en búsqueda, sin certezas, pero con la compañía del grupo con el cual van reelaborando su práctica.

En ese mismo orden de dirección, en la esfera del mundo micro - social van 
surgiendo los fundamentos de las nuevas colectividades que dan forma a la utopía de estos tiempos en educación, y él y ella participan sabiendo que es un campo en construcción, y ambos son parte de ello, haciendo real y concreta la idea de "vida buena", planteada por nuestros grupos originarios como una manera de mantener la unidad de la naturaleza y la cultura. Por ello, la propuesta no es formar científicos, es construir una cultura ciudadana y democrática en ciencia, tecnología e innovación para estos tiempos de un mundo construido sobre el conocimiento, la tecnología, la información y la comunicación.

Este ejercicio pedagógico aparece como fundamento de una nueva forma de lo público que, a través de la idea de justicia educativa y justicia curricular, trabaja por construir sociedades más justas y menos desiguales. Y si después de esto los niños, niñas y jóvenes optan por ser científicos lo serán también de otra manera, como parte de la búsqueda iniciada desde la propuesta metodológica que contiene como valores fundamentales esas capacidades de lo humano (cognitivas, afectivas, valorativas y de acción) sobre las cuales se fundamenta la investigación como estrategia pedagógica.

El punto de partida para buscar caminos alternativos en educación desde una perspectiva crítica se da a partir de un triple reconocimiento: Primero, de cómo, derivado de la revolución científico-técnica en marcha, se asiste a una reconfiguración del saber escolar de la modernidad, lo cual se debe convertir en una oportunidad para ir más allá de una simple modernización de la educación e intentar transformar las relaciones de poder que controlan y dominan con propuestas pedagógicas y metodológicas inclusivas que muestren diseños concretos, más allá de la simple denuncia y crítica general a esa modernización.

El segundo hecho por ser reconocido es el lugar que el trabajo inmaterial (trabajo intelectual) ha tomado en esta sociedad, al convertirse en un generador de valor $\mathrm{y}$, por lo tanto, elemento central para construir el capitalismo de este tiempo, lo cual convierte a actores que trabajan con el conocimiento, la tecnología, la información y la comunicación en sujetos claves de los nuevos procesos de conformación de esta sociedad, alcanzando los educadores de todos los tipos un carácter que organiza y da forma a la base y fundamento de estas modificaciones sociales, (Mejía, 2011).

Es un ejercicio que va a requerir a todo educador, reconocerse y construirse como actor en estas nuevas condiciones, lo cual va a exigir un replanteamiento de las formas anteriores y la consabida modificación de su nueva condición, así como de las transformaciones institucionales en las cuales desarrolla su quehacer. En ese sentido, su subjetividad y los escenarios de su acción se convierten en campos resemantizados y, por lo tanto, en disputa para construir un proyecto de emancipación o de simple modernización al servicio de los grupos dominantes en la sociedad. Es allí donde el educador pone en escena su capacidad humana al servicio de intereses precisos y concretos, (Cetrulo, 2001).

El tercer elemento por reconocer desde una perspectiva crítica es cómo uno de los aspectos centrales en la configuración de un trabajo inmaterial es la investigación, la que da forma a este nuevo proyecto de control del capital, siendo este uno de los trabajos de este tiempo que más valor genera, por lo tanto, es clave en la modalidad actual de acumulación. Allí se reconoce el lugar de ella, como un factor 
clave en la configuración de la revolución época en la cual nos encontramos.

A la vez que ha generado estas condiciones nuevas en la sociedad, se ha constituido a sí misma como campo de conocimiento, generando saberes y aproximaciones diversas desde y hacia ella, y en muchas ocasiones, con sus particularidades especificidades. En ese sentido, pensar la investigación desde las corrientes educativas criticas significa una lucha teórico-práctica por la manera como sus presupuestos sobre el conocimiento, la ciencia, su epistemología, la cultura, lo humano y los grupos sociales enmarcan una acción que durante cuatrocientos años ha sido señalada como objetiva, y que no solo ha construido una forma de ella, sino que también ha ayudado a generar formas de poder que en la sociedad han servido para el control y la gestación de desigualdades, y en estos tiempos, nuevas formas de acumulación y dominación, (Ortega, 2009).

Dicho en otras palabras, se requiere reconocer la categoría de la investigación en la sociedad, a la vez que se develan los intereses presentes. En su práctica forja un vistazo crítico, que en coherencia con los tiempos presentes se ve obligada no solo a la denuncia, sino también a apropiarse de ella como una realidad muy importante en esta época para construir propuestas que a la vez que promuevan su apropiación práctica crítica, sirva no solo para modernizar, sino, ante todo, para transformar contextos, culturas, epistemologías, y redirigir sus escenarios de poder para construir subjetividades y ciudadanías que reconociendo el lugar de lo local se abran a un tiempo-espacio global y a las nuevas mediaciones científico-tecnológicas de este tiempo para hacer concreta la construcción sociedades más democráticas, justas y humanas, así como la necesidad de un planteamiento sobre ella en cualquier proyecto emancipador.

En América Latina, así como en grupos subalternos en el mundo del Norte y en diferentes actores críticos de otros continentes, se ha desarrollado, en la perspectiva de los acumulados del paradigma latinoamericano, una concepción de trabajo educativo que se caracteriza por ser una acción política en la esfera de la educación; ella busca transformar las condiciones de control, dominio y formas de sujeción de actores, comunidades e instituciones. Para lograrlo busca generar conciencia crítica y dinámicas sociales que impulsen que los grupos construyan formas de asociación y organización que los convierte en sujetos colectivos, constructores de su historia, (Gadotti, 2003).

La investigación como estrategia pedagógica ha tomado de la educación popular algunos de sus elementos básicos y los ha convertido en ejes de su propuesta, diferenciándose de otros acercamientos de pedagogías fundadas en la investigación, a la vez que realiza un proceso de construcción colectiva de su propuesta, donde incorpora niños, niñas, jóvenes, maestras, maestros, asesores, dándole forma a una particular manera de generar procesos de educación popular con estos grupos. Por ello su punto de partida es la realidad de estos grupos y sus saberes, para elaborarlos a partir del proceso investigativo.

En los departamentos y municipios, los actores optan por desarrollar la propuesta; es decir, constituyen redes (sociales, políticas, de actores institucionales) que hacen real esa búsqueda y se coordinan para concretarla en los diferentes territorios y espacios de sus respectivas localidades. Estas redes han reconocido 
la necesidad de transformar los procesos de la educación, de la relación entre adultos y niños y niñas, así como de las relaciones sociales que se mediatizan en estas prácticas.

Por ello, esa opción se desarrolla en el contexto, con las particularidades de la cultura, desde las subjetividades constituidas. Todas ellas en una relación: organizaciones sociales, subjetividades, prácticas sociales, dinamizando un escenario de individuación, en donde la integralidad de lo humano (razón, emoción, acción, intereses) debe ponerse en juego para reconocer que nos hacemos humanos en la diferencia y en una interacción conflictiva con los otros y sus realidades.

Un principio de la educación popular, en la cual la realidad se conoce para transformarla transformándose a sí mismo, y esto desde un horizonte ético que busca develar y enfrentar la segregación, la exclusión y formas de control y dominación que se produce por las múltiples manifestaciones del poder en nuestra sociedad: políticas, económicas, sociales, étnicas, en el conocimiento, de subjetividades, de género y en el ejercicio de la práctica educativa y pedagógica; construir un espíritu de emancipación humana, para no desarrollar procesos de poder que controlan ni dominan, ni permitir que otros lo realicen en su propia vida, (Torres, 2007).

La IEP postula la existencia de saberes que se mueven más en la experiencia y las actuaciones de los seres humanos, y de conocimientos que están más dados en la esfera de las disciplinas; de esta forma, postula la existencia de racionalidades diferentes a la eurocéntricanorteamericana (Mignolo, 2003). Las cuales también deben ser reconocidas por el ejercicio educativo, haciendo real la interculturalidad y la negociación cultural; este ejercicio debe darle cabida a las razones más allá de la "razón universal", para hacer visible el reconocimiento del otro y de lo otro diferente a aquello que es postulado como universal, fruto de la lógica de control y de poder, visibilizar en los diferentes su saber, su historia, su cultura, y, cuando sea el caso, reconocerlos como epistemes negadas o invisibilidades. Va a ser el ejercicio de reconocer los contextos como lugares de saber. Por ello, busca devolver al acto educativo diferencia, heterogeneidad y multiplicidad fundadas en una diversidad cultural, social y cognitiva.

Desde ahí, lo que se construye en cualquier acción educativa son relaciones sociales, en las cuales se manifiestan bajo formas pedagógicas aquello que pensamos de la sociedad. Por eso, el papel del educador es ser enseñante - aprendiente, reconociendo la manera como se forma a la vez que va formando. En este sentido, el papel del formando es activo y también forma a sus adultos acompañantes, a la vez que va aprendiendo, gestándose unas relaciones educativas desde lo diferente en la interculturalidad, (De Souza, 2001).

En el respeto y el reconocimiento de los aportes de cada uno. Por ello en la IEP, maestro y maestra se convierten en acompañantes co-investigadores, transformando su rol tradicional y reconociéndose como aprendientes en el proceso. En el caso de la IEP, se parte de la pregunta del sentido común de los niños, niñas y jóvenes para reelaborarla en la perspectiva de la educación popular, que reconoce la existencia de saberes comunes y elaborados así en la negociación con los conocimientos disciplinares, y de las maneras como se correlacionan en la negociación cultural (Mariño, 2010), lo 
cual muestra la manera en que se van dando estas transformaciones, a través del aprendizaje colaborativo, en el cual intervienen niños y adultos. En el sentido de Vigotsky, toda función aparece en dos planos: en lo social (interpsíquico) y en lo psicológico (intrapsíquico).

La idea de aprendizaje es retomada también de este enfoque y reelaborada para Vigotsky: "El aprendizaje es un aspecto universal y necesario del proceso de desarrollo culturalmente organizado y específicamente humanos de las funciones sicológicas"; es decir, este, organizado pedagógicamente, se convierte en desarrollo mental y sigue generando procesos evolutivos que no se podrían dar sin él. Es allí donde las relaciones entre el sujeto y el mediador son activas y recíprocas, en la internalización se da el conocimiento. En ese sentido, el aprendizaje es una actividad propia diferenciada, por ello no se puede hacer equivalente a desarrollo.

\section{Metodología}

\section{Diseño}

La metodología del proyecto de investigación se realizó a partir de los recorridos de las trayectorias de indagación, basados en la integración de la IEP apoyada en TIC al aula, articulado al plan de estudios, desde el área de sociales se estructuraron temáticas relacionadas con la violencia sexual y estrategias preventivas del cuidado del cuerpo, la finalidad de este proceso investigativo, es generar una trasformación social y educativa a través del aprendizaje en contexto, involucrando intereses, motivaciones y realidades de los estudiantes para generar conocimiento científico, donde se establecieron cinco (5) momen- tos que especifican en la ejecución y las metas trazadas para darle solución a la pregunta problema planteada.

\section{Participantes}

Se tomó una muestra de cuarenta (40) estudiantes de la IED Francisco de Paula Santander, sede Simón Bolívar, pertenecientes al grado segundo de primaria, con edades que oscilaron entre los siete (7) y (9) años, en cuanto a la información sociodemográfica, los participantes del estudio pertenecen a estratos muy bajos y han reportado situaciones de disfuncionalidad familiar, algunos no viven con sus padres, sino con familiares cercanos, por lo cual se buscó implementar estrategias preventivas desde la escuela.

\section{Técnicas e instrumentos}

En cuanto a los instrumentos de recolección se utilizó el diario de campo, este es un instrumento que ayuda al investigador a inmiscuirse de forma total en el ambiente con el cual se va a encontrar, es un proceso de hechos, recolección de datos y análisis. El investigador entiende a los participantes y no se dedica únicamente a registrar "hechos". El diario de campo es parte de un registro cronológico que se desarrolla mediante la sesión compartida con los infantes, es donde se describe detalladamente los aspectos positivos como negativos colocando significado a cada uno de ellos como un recurso de mejoras en todas las áreas con el grupo a trabajar considerando los recursos manejados y utilizados. Un cuaderno donde se va a descubrir la manera de expresar y escribir frente a la relación que se tiene con los infantes, es un momento de no cohibir lo vivido y las experiencias obtenidas. 


\section{Procedimiento}

A continuación, se presentan los diferentes momentos, entre estos:

- Momento (1): selección y análisis de la población sujeto de estudio.

- Momento (2): integración de la temática mediante la IEP al aula de clases, se integraron grupos estudiantiles para seleccionar problemáticas relacionadas con la violencia sexual. Los estudiantes se plantearon preguntas y con el acompañamiento del docente realizaron el proceso de indagación mediante herramientas virtuales y entrevistas a la comunidad.

- Momento (3): socialización de los resultados de las indagaciones de estudiantes en una feria institucional dirigida por los docentes a cargo.

- Momento (4): análisis de los resultados de la implementación de la estrategia a través de la observación y diario de campo.

- Momento (5): propagación y divulgación de los resultados.

\section{Resultados}

De acuerdo a las técnicas utilizadas y a los datos obtenidos en los resultados al finalizar el estudio se observó mediante las diferentes actividades elaboradas que existe poca orientación por parte de los padres de familia en educación sexual, teniendo en cuenta que los niños en las edades comprendidas en el estudio se encuentran en un proceso de exploración del cuerpo se hace necesario ofrecerles herramientas para identificar los posibles riesgos y saber cómo actuar frente a una situación de peligro. La investigación permitió que fueran ellos quienes construyeran su propio conocimiento, indagando por todas las inquietudes que presentaban frente al tema, donde los docentes fueron orientadores en esa construcción.

Las actividades diseñadas se realizaron teniendo en cuenta la investigación como estrategia pedagógica dentro del aula de clases apoyada en las TIC con el fin de identificar situaciones de riesgo en las que se pueda presentar un posible abuso. Los estudiantes buscaron con ayuda del docente estadísticas, casos en YouTube y realizaron entrevistas dirigidas a la comunidad donde recolectaron amplia información sobre el tema. Además, los resultados dejaron en evidencia que la población objeto de estudio no contaba con conocimientos previos sobre el tema de investigación, en el proyecto se establece la necesidad de incluir la educación sexual al aula desde la edad preescolar como recomendación a futuros estudios en la institución educativa.

Además, la institución educativa, dentro de su plan de estudios no contempla proyectos relacionados con la sexualidad aplicada a las actividades extracurriculares, donde sería importante contar con la participación de los padres de familia en la elaboración de actividades que permitan ser más expresivos con sus hijos, generando en la familia comunicación, apoyo, relaciones afectivas y colaboración mutua.

Como estrategia de propagación se realizó un evento informativo donde los niños investigadores expusieron ante toda la comunidad educativa el trabajo realizado, así como también solicitaron a docentes y alumnos de otras sedes unirse a esta iniciativa. Además, los grupos deben compartir sus resultados en eventos a través de diferentes 
medios de comunicación. Los productos de la investigación se divulgan en los espacios virtuales y físicos del programa Ciclón consolidando la comunidad de práctica saber, aprendizaje, conocimiento y trasformación: en espacios físicos como: asesorías de línea temática, eventos de apropiación, ferias, encuentros el maestro tiene la palabra, comités subregionales, espacios de formación de maestros y publicaciones impresas.

\section{Conclusiones}

En síntesis, la revisión realizada sobre las consecuencias psicológicas a corto plazo de la prevención de la violencia sexual constata su frecuente presencia y su diversidad, así como la dificultad que implica el estudio de este tema, con múltiples problemas metodológicos que impiden avanzar en el conocimiento del estado psicológico de las víctimas, dificultando el desarrollo de programas de tratamiento eficaces y específicos, (Paolucci, Genuis y Violato, 2011).

En ese sentido, los procesos de educación formal deben promover un aprendizaje y valoración de la sensibilidad, de la paz, de la solidaridad, de igual forma, mediante una prevención de la violencia sexual que es posible cuando los estudiantes se encuentran informados y conocen los riesgos a los que se enfrentan en la ruta de atención a una situación de abuso, sin embargo, en el estudio se encontró que desde la familia no se enseñan estrategias preventivas, por lo cual es necesario tomar la iniciativa desde la escuela.

La investigación como estrategia pedagógica permitió desde la academia formar al estudiante basado en un enfoque basado en estrategias preventivas donde si direccione a un rol activo en el proceso de construcción del conocimiento mediante técnicas propias de las corrientes investigativas, aportando una formación integral del ser, donde no solo se genere un nuevo conocimiento sino que los estudiantes logren apropiarse del mismo y socializarlo, desarrollando competencias necesarias para el desarrollo intelectual como, pensamiento crítico, búsqueda de información, competencias orales y comunicativas, análisis de resultados y entrevistas, enmarcadas en la formación de cultura ciudadana en ciencia, tecnología e innovación.

\section{Referencias bibliográficas}

Almonte, C., Insunza, C., y Ruiz, C. (2002). Abuso sexual en niños y adolescentes de ambos sexos. Revista chilena de neuropsiquiatría, 40(1), 22-30.

Anceschi, A. (2009). La violenza familiare: aspetti penali, civil e criminologici. Torino: G. Giappichelli.

Bravo, H. (2008). Estrategias pedagógicas. Córdoba. Universidad del Sinú.

Cetrulo, R. (2001). Alternativas para una acción transformadora: Educación popular, ciencias y política. Montevideo. Ediciones Trilce. Instituto del Hombre.

De Souza, J. (2001). Recife: Edições Bagaço.

Gadotti, M. (2003). Perspectivas actuales de la educación. Buenos Aires: Siglo XXI.

Gómez, S., \& Cobo, D. (2013). Abuso sexual en niños. Revista Gastrohnup. Volumen 15 Número 1 Suplemento 1.

Hernández, R., Fernández, C., \& Baptista, P. (2010). Metodología de la investigación. México. Editorial Mc Graw Hill. 
Mariño, G. (2010). El diálogo en la educación de jóvenes y adultos. Dos propuestas pedagógicas para implementarlo. En: El taller dialógico/la recuperación de experiencias laborales. Bogotá: OEI.

Mejía, M. R. y Manjarrés M. H. (2010). Las pedagogías fundadas en la investigación. Búsquedas en la reconfiguración de la educación +Revista Internacional Magisterio 42: 16-26. Colombia.

Mejía, M. (2011). Las escuelas de la globalización. El conflicto por su reconfiguración. Bogotá: Desde Abajo.

Meresman, S. (2011). Es parte de la vida. Material de apoyo sobre educación sexual y discapacidad para compartir en familia.

Mignolo, W. (2003). Capitalismo y geopolítica del conocimiento. Buenos Aires: Paidós

Ministerio de Educación en Colombia. Recuperado de http://www.mineducacion.gov.co/1621/article- 173947. html

Miranda, M. (2014). Familia y emociones: un reto pedagógico en la búsqueda de la salud en México. Revista Internacional de Educación y Aprendizaje, 2.
Morillo, B; Montero, L; Colmenares, Z. (2012). Conocimiento de los padres en el abuso sexual infantil. Revista electrónica, Universidad de Carabobo de Venezuela,

OMS, (2016). Violencia sexual en Latinoamérica y el Caribe: Análisis de datos secundarios.

Ortega, P. (2009). Sujetos y prácticas de la pedagogía crítica. Bogotá: El Búho.

Paolucci, E. O., Genuis, M. L. y Violato, C. (2011). A meta-analysis of the published research on the effects of child sexual abuse. The Journal of Psychology, 135 (1), 17-36

Pereda, N. (2010). Consecuencias psicológicas a largo plazo del abuso sexual infantil. Revista Electrónica, Vol. 31 No.2.

Pozo, J. y. (2006). Aprender y enseñar ciencia. Madrid: Morata.

Torres, A. (2007). La educación popular. Trayectoria y actualidad. Bogotá: El Búho.

UNICEF. Abuso sexual a niños, niñas y adolescentes. Guía conceptual. $72 \mathrm{p}$, 24-25. Primera edición, junio de 2013 Disponible en: http://www.unicef.org/ argentina/spanish/educacion_Abuso_ Sexual_170713.pdfwww.unicef.org/ argentina/spanish/educacion_Abuso_Sexual_170713.pdf $\square$. Consultado el 11 de febrero 2014. 